\title{
BERYLLIUM PRESSURE BAR HAVING A SUBMICROSECOND RISETIME
}

\author{
Ieuan.R. Jones
}

\section{Abstract}

The theoretical response of a pressure bar gauge to step-pressure loading is examined experimentally. Theory predicts that a pressure bar constructed from beryllium should have a risetime substantially faster than that of any previous pressure bar gauge described in the literature. Such a gauge was constructed and subjected to step-pressure loading; the time taken for the output signal to increase from $10 \%$ to $90 \%$ of its final steady value is 0.54 usec. 
Introduction

Measurement of pressures varying on the microsecond time scale has become increasingly important in recent years. One of the most satisfactory methods of measuring such pressures is the pressure bar technique. Originally devised by Hopkinson ${ }^{1}$, it was suhsequently developed into a more sophisticated method by Davies ${ }^{2}$ during World War II. During the last 20 years or so, members of the Department of Physics, University College of Wales, Aberystwyth, have made extensive use of this technique in experiments involving detonation waves $^{3}$, shock waves ${ }^{4}$ and cavitation in liquids ${ }^{5}$. The pressure bar technique has of late been applied to studies in plasma physics ${ }^{6-9}$.

In essence the method is as follows. The unknown pressure is applied to one end of a circular cylinder of elastic material. This initiates propagation of an elastic stress pulse composed of longitudinal waves along the bar; from a study of this pulse at some station along the length of the bar, the variation of the applied pressure with time is deduced. There are a number of ways of detecting the stress pulse propagating in the bar but undoubtedly the most satisfactory is that reported by Edwards ${ }^{10}$ in which a piezoelectric disc is sandwiched in the bar. Iongitudinal stress waves travelling along the bar traverse the disc and the charge consequently liberated at its faces is measured in a convenient manner. A general review of the pressure bar technique which includes a discussion of its inherent limitations may be found in Ref. 11. Calibration techniques are also discussed in this reference.

The main advantages of the bar gauge over other types of pressure gauges are the following. It is essentially an aperiodic device and its output signal is relatively free from parasitic oscillations arising from excitation of natural frequencies of the gauge. Its high frequency response is superior to that of most other gauges and is adequate for most, but not all, purposes. The acoustic delaying of the pressure signal in the first section of the pressure bar allows one to make readily pressure measurements in an environment of large electrical interference such as is usually encountered in plasma physics experiments. 
The response of a pressure bar to step-pressure loading

It is convenient to discuss the high frequency response of a pressure bar in terms of its response to the application at its measuring end of an uniform pressure having a step function time dependence. Clearly, a characteristic quantity such as the risetime of the output signal is intimately connected with the high frequency response of the bar.

Theoretical treatments of the problem of the shock-loading of a bar have been given by Folk, Fox, Shook and Curtis ${ }^{12}$ and by Rosenfeld and Miklowitz ${ }^{13}$. The formal solution for the displacement field is in terms of integrals which cannot be evaluated by straightforward methods. If, however, only large values of $z_{0}$ are considered, where $z_{0}$ is the distance along the bar from the stressed end to the point of observation, then expressions for the displacements may be developed by looking at appropriate domains in the frequency spectrum. In particular, it can be shown from the above papers that the relative axial stress, for short times following the arrival of the stress pulse at the observation point and in the limit of large $z_{0}$, is given by the following expression.

$$
\begin{aligned}
& \text { Relative axial stress }=\frac{\sigma_{Z Z}}{\sigma_{Z Z}(\text { final value })} \\
& =\frac{1}{3}+\int_{0}^{B} A i(-x) d x \\
& \text { where } B=\frac{t^{\prime}}{z_{0}^{1 / 3}}\left[\frac{4 c_{0}^{3}}{3 y^{2} a^{2}}\right]^{1 / 3}, A i(x) \text { is the Airy integral, } \\
& t^{\prime}=\left(t-\frac{z_{0}}{c_{0}}\right),
\end{aligned}
$$

$t$ is time measured from the instant at which pressure is applied to the bar, a is the radius of the bar, $\boldsymbol{\mathcal { N }}$ is Poisson's ratio for the bar materia and $c_{0}$ is the bar velocity $(=\sqrt{\mathbf{P}}$, where $\mathrm{Y}$ is Young's modulus and $\boldsymbol{p}$ is
the density of the bar material). 
The validity of the above expression has been investigated experimentally by Fox and Curtis ${ }^{14}$ and by Edwards et al ${ }^{4}$. They showed that for values of $z_{0}$ satisfying $z_{0} / 2 a>20$, the detailed evolution of the relative stress with time (for early times) was in good agreement with theory. This asymptotic solution also predicts that for large values of $z_{0}$, the risetime varies as $z_{0}{ }^{1 / 3}$. Eawards et al 4 found that this dependence was obeyed in the range $0.125 \leqslant 20 / 2 a \leqslant 65$.

Both the above mentioned experimental studies were carried out with large diameter ( $\boldsymbol{\sim}_{3} \mathrm{~cm}$ ) bars. Consequently the dispersive oscillations of the bars were of fairly low frequencies (typically about $50 \mathrm{Kc} / \mathrm{s}$ ). Pressure bars having good high frequency response must, however, of necessity be constructed from small diameter bars. For example, pressure bar gauges which have recently been reported in the literature have had diameters of between 1 and $3 \mathrm{~mm}$. For these gauges, the bar oscillations have a frequency of between 0.5 and $I \mathrm{Mc} / \mathrm{s}$. The question of the effect of sound attenuation on the risetime of the bar gauge could now become an important one; it should be remarked that dissipation is not included in the theory on which $\mathrm{Eq}$. (I) is based. This being so, it was decided to examine experimentally the validity of $\mathrm{Iq}$. (l) for the case of a small diameter bar.

A $0.3 \mathrm{~cm}$ diameter duralumin pressure bar was located with its measuring end flush with the end plate of a conventioral diaphragm shock tube. In this position the bar experiences a good approximation to step-pressure loading due to the reflection of the incident shock wave at the end of the expansion chamber. The stress pulse propagating along the bar was detected by means of a FZT-4 polarized ferroelectric disc sandwiched into the bar. Measurements were carried out for values of $z_{0}$ varying between $0.85 \mathrm{~cm}$ and $34.95 \mathrm{~cm}$ i.e. in the range $2.83 \leqslant \mathrm{Z} / 2 \mathrm{a} \leqslant 116.3$. Typical examples of the oscillograms obtained in this study are shown in Fig. 1. The upper trace shows that on arrival at the observation station, the stress increases to a value which is greater than the final value and then begins to oscillate about the final value with a progressively decreasing period and amplitude. The lower trace merely shows the head of the pulse on an expanded time scale ( 1 usec/div). 
For a pressure bar of given material and radius, numerical integration of the integral in $\mathrm{Eq}$. (1) yields the relative stress as a function of the parameter $t / z_{0}^{1 / 3}$. Such a theoretical curve was computed for the pressure bar used in these measurements; it is shown in Fig. 2. The constants used in this calculation were :

$$
\begin{aligned}
& a=0.15 \mathrm{~cm} \\
& c_{0}=0.511 \times 10^{6} \mathrm{~cm} / \mathrm{sec} \quad \text { (measured) } \\
& \boldsymbol{\nu}=0.33 \quad \text { (assumed) }
\end{aligned}
$$

If the theory is valid, then all the experimental data obtained with different values of $z_{0}$ should, when plotted as a function of $\mathrm{t} 1 / \mathrm{z}_{0}^{1 / 3}$, reduce to a single curve, namely the theoretically derived one.

The experimental results were reduced as follows. The experimentally determined stress-time curve for a given value of $z_{0}$ was normalized with respect to the first maximum of the theoretical curve and the zero point of the parameter $t / / z_{0}^{1 / 3}$ was chosen by fitting by eye the initial portions of the curves. The result of such a procedure for two values of $z_{0}\left(z_{0} / 2 a=21.4\right.$ and 116.3$)$ is shown in Fig. 2. The present measurements show that the theory is applicable even when the absolute time scale of the phenomenon is about a factor ten shorter than for that reported in Refs. 4 and 14.

A quantity which is of considerable interest and which is amenable to comparison with theoretical prediction in a completely unambiguous manner is the risetime of the output signal from the bar. For our present purposes we shall define the risetime, $\tau_{I}$, to be the time taken for the signal to increase from $10 \%$ to $90 \%$ of its maximum value. The approximate expression of $\mathrm{Eq}$. (1) predicts that for large $z_{0}$ the risetime varies as $z_{0}^{1 / 3}$. In particular, for the pressure bar used in these measurements,

$$
\begin{aligned}
\tau_{1} & =2.28 \boldsymbol{\nu}^{2 / 3}\left(\frac{z_{0}}{a}\right)^{1 / 3}\left(\frac{a}{c_{0}}\right) \\
& =0.60 z_{0}^{1 / 3}
\end{aligned}
$$


where the units of time and length are usec and cm respective-y. The results of experiment together with the appropriate theoretical dependence are shown in Fig. 3. Each experimental point is the mean of at least three measurements and the error flags refer to the root mean square error. The discrepancy between theory and experiment increases with decreasing $Z_{0}$ but even for the smallest value of $z_{0}$ used in these measurements $\left(z_{0} / 2 a=2.83\right)$, theory predicts a risetime which is only $20 \%$ faster than that actually observed. It is not known whether this discrepancy between theory and experiment is directly attribleble to a frequency dependent bar effect or, on the other hand, is a result of the pressure input to the gauge not being truly step-functional in its time-dependence.

On the basis of the present measurements we may safely use the relationship expressed in Eq.(2) as a guide to the design of small diameter pressure bars. It is seen that for a pressure bar of given dimensions, the risetime is faster if materials are used which have small values of Poisson's ratio but large values of bar velocity. The most favourable material appears to be beryllium for which Poisson's ratio is 0.05 and the bar velocity is $1.29 \mathrm{~cm} / \mu \mathrm{sec}$. The use of beryllium as a pressure bar material was first suggested by Baganoff ${ }^{15}$ but he put aside the idea on the grounds that a "piezoelectric material with properties matching the appropriate physical properties of beryliium" was not available. Presumably, in the above quotation Baganoff is referring to the acoustic matching of the piezoelectric disc and beryliium. However, piezoelectric ceramics having acoustic impedances close to that of beryllium are available and, moreover, it may be shown that, provided the wavelengths (based on the velocity in the piezoelectric material) of the elastic waves concerned in the propagation of the stress pulse are large compared with the thickness of the piezoelectric disc, the question of acoustic matching is not an important one. The performance of a pressure bar constructed from beryllium is described in the next section of this report. 
Beryllium pressure bar

A pressure bar was constructed from a beryllium bar of $0.3 \mathrm{~cm}$ diameter and $20 \mathrm{~cm}$ length. The bar was cut into two equal lengths and a PZT-4 polarized ferroelectric disc of $0.3 \mathrm{~cm}$ diameter and $0.05 \mathrm{~cm}$ thickness was cemented between the two pieces. The potential user is warned of the health hazard presented by beryliium; the usual safety precautions should be strictly adhered to when handing this material.

Fig. 4 shows the oscillograms which were obtained when the bar was subjected to step-pressure loading. The negative going signal observed in the upper trace of this figure at approximately 15 usec after the initial rise in signal is due to the reflected pulse of tension from the far end of the pressure bar. The lower trace of Fig. I shows the head of the pulse on an expanded time scale $(0.5 \mu \mathrm{sec} / \mathrm{div})$.

The oscillations which occur immediately after the initial rise in signal could not be identified with dispersive oscillations in the beryllium bar. It was suspected that these were radial oscillations of the piezoelectric disc itself. In order to test this hypothesis, a $0.2 \mathrm{~cm}$ diameter, $0.03 \mathrm{~cm}$ thick, PZT-5 polarized ferroelectric disc was substituted for the $0.3 \mathrm{~cm}$ diameter disc and the oscillogram shown in Fig. 5 was obtained. After taking into account the fact that the transducers were made of different materials, it was found that the ratio of the frequencies did indeed correspond, within the limit of experimental error, to the inverse ratio of the disc radii. Furthermore, each frequency corresponded to the appropriate disc dimension.

The exact nature of the mechanism responsible for the excitation of the radial oscillations in the discs is not known. There seems, however, to be some evidence that the amplitude of this oscillation depends on the cement bond joining the piezoelectric element to the berylitum bar. If the cement joints are bad, and by bad we mean that on subsequent inspection the cemont layers are found to have air 
bubbles entrapped within them, then signal outputs similar to the ones shown in Fig. 6 are obtained. No effort was made to perfect an optimum cementing technique. The usual procedure was a trial and error one involving the cutting of the ends of the bar square with the axis, the polishing of the mating faces with polishing cloth and, finally, the cementing of the piezoelectric element into place with household Araldite which was rendered conducting by the addition of silver powder. The joints were held together in a V-block under moderate pressure for the duration of the setting process.

If we assume that the pressure acting on the stressed end of the beryllium pressure bar has truly a step-function time dependence, then the risetime of this bar can be measured from the oscillogram shown in Fig. 4. The time, $\tau_{2}$, taken for the output signal to increase from $10 \%$ to $90 \%$ of its steady final value is measured to be $0.54 \mathrm{ksec}$. The theoretical risetime is calculated to be $0.125 \mu \mathrm{sec}$. In view of the fact that the transit time across the PZT-4 disc is $0.143 \mathrm{ksec}$, there seems to be no doubt that some of the discrepancy between the measured and theoretical risetime arises from the finite thickness of the transducer and also from the acoustic mismatch between the beryllium and the PZT-4. The cement bonds can also possibly contribute to a longer risetime. We wish to emphasize, nevertheless, that the pressure bar which has been tested possesses a substantially faster risetime than any other hitherto reported. The intrinsic high frequency limit of the berylitum bar has not been reached and possibly even faster risetimes could result from the use of thinner ferroelectric discs and greater care with the cementing procedure.

\section{Acknowledgments}

The author would like to express his appreciation to Dr. F. Troyon for many beneficial discussions and to Mr. Ripper for his competent technical assistance. This study was financed by the Swiss National Science Foundation. 
1. B. Hopkinson, Phil. Trans. Roy. Soc. (London) A 213, 437 (1914)

2. R.M. Davies, Phil. Trans. Roy. Soc. (London) A 240, 375 (1948)

3. D.H. Edwards, G.T. Hilliams and J.C. Breeze, J. Fluid Mech. 6 , 497 (1959)

4. D.H. Edwards, L. Davies and T.R. Lawrence, J. Sci. Instr. 41, $609(1964)$

5. I.I. Jones and D.H. Edwards, J. Fluid Mech. I, 596 (1960)

6. M.O. Stern and I.N. Dacus, Rev. Sci. Instr. 32, 140 (1961)

7. T.K. Allen, R.W.P. McWhirter and I.J. Spalding, Nuclear Fusion, 1962 Supplement, Part I, 67

8. K. Büchl, Z. Naturforschung 19a, 690 (1964)

9. R.M. Measures, Ph. D. Thesis, University of London (1964) (to be published)

10. D.H. Edwards, J. Sci. Instr. 35, 346 (1958)

11. I.R. Jones, Report No TDR-594 (1208-01) TR-3, June 1961 Aerospace Corporation, $\mathbb{T} 1$ Segundo, Calif.

12. I. Folk, G. Fox, C.A. Shook and C.W.Curtis, J. Acoust. Soc. Am. 30, 552 (1958)

13. R.I. Rosenfeld and J. Miklowitz, J. Appl. Mech. 32, 290 (1965)

14. G. Fox and C.W. Curtis, J. Acoust. Soc. Am. 20, 559 (1958)

15. D. Baganoff, Rev. Sci. Instr. 35, 288 (1964) 
Fig. I Oscillograms of the stress observed in a $0.3 \mathrm{~cm}$ diameter duralumin bar. The stress is generated by the reflection of a shock wave at one end of the bar and the point of observation is $34.95 \mathrm{~cm}$ from the stressed end. Time scales : Upper trace, $5 \mathrm{usec} / \mathrm{div}$ Lower trace, $\quad 1 \mu \mathrm{sec} / \mathrm{div}$ (1Time marks every 1 ksec)

Fig. 2 Comparison of observed and predicted shape for head of the pulse. $t^{\prime}=t-z_{0} / c_{0}$

Fig. 3 Comparison of observed and predicted dependence of risetime on the distance, $Z_{0}$, of the observation point from the stressed end.

Fig. 4 Oscillograms of the signal obtained from a $0.3 \mathrm{~cm}$ diameter beryllium pressure bar when the measuring end is stressed by means of a reflected shock wave. $\Lambda 0.3 \mathrm{~cm}$ diameter, $0.05 \mathrm{~cm}$ thick, polarized ferroelectric (PZT-4) disc is located $10.0 \mathrm{~cm}$ from the stressed end. Time scales : Upper trace, $5 \mu \mathrm{sec} / \mathrm{div}$ Lower trace, $0,5 \mu \mathrm{sec} / \mathrm{div}$

Fig. 5 Oscillograms of the signal obtained from a berylium pressure bar when the measuring end is stressed by means of a reflected shock wave. The diameter of the bar is $0.3 \mathrm{~cm}$ but the diameter of the $0.03 \mathrm{~cm}$ thick, polarized ferroelectric (PZT-5) disc located $10.0 \mathrm{~cm}$ from the stressed end is $0.2 \mathrm{~cm}$.

Time scales : Upper trace, 5 usec/div Lower trace, $0.5 \mu \mathrm{sec} / \mathrm{div}$

Pig. 6 Bxamples of output signal obtained from the beryliium pressure bar when the ferroelectric disc is badly cemented into the bar Time scales : Upper trace, $5 \mu \mathrm{sec} / \mathrm{div}$ Iower trace, $5 \mu \mathrm{sec} / \mathrm{div}$

(The signal polarity is reversed for the lower trace.) 


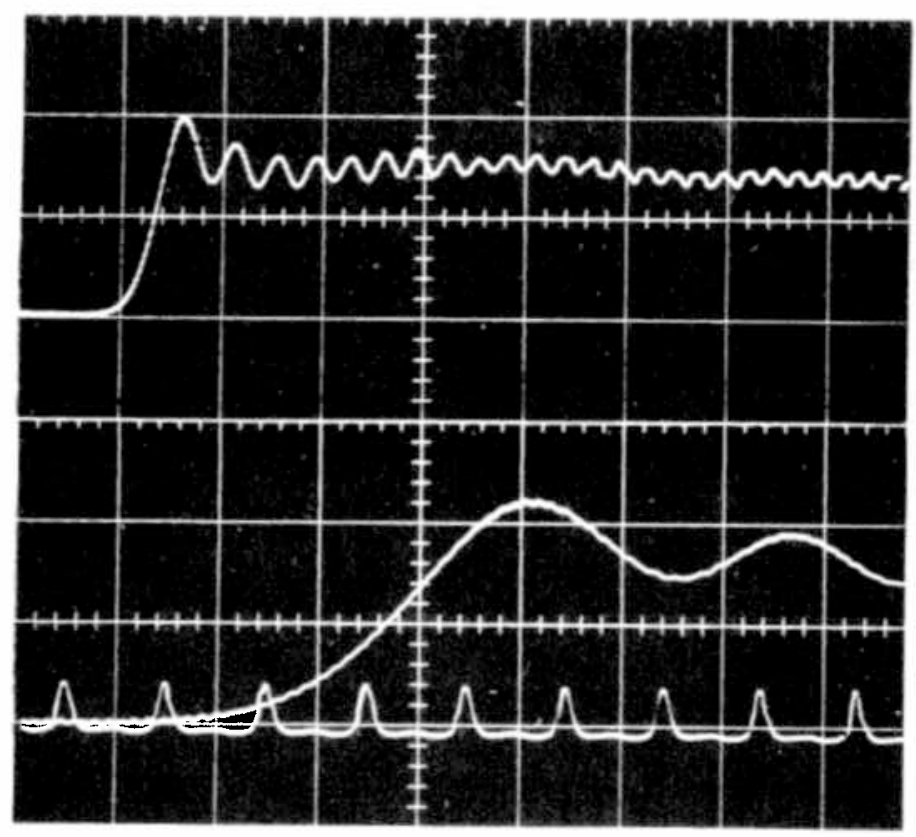

\section{Fig. 1}

Oscillograms of the stress observed in a $0.3 \mathrm{~cm}$ diameter duralumin bar. The stress is generated by the reflection of a shock wave at one end of the bar and the point of observation is $34.95 \mathrm{~cm}$ from the stressed end.

Time scales : Upper trace, $5 \mathrm{\mu sec/div}$ Lower trace, 1 usec/div

(Time marks every 1 usec) 


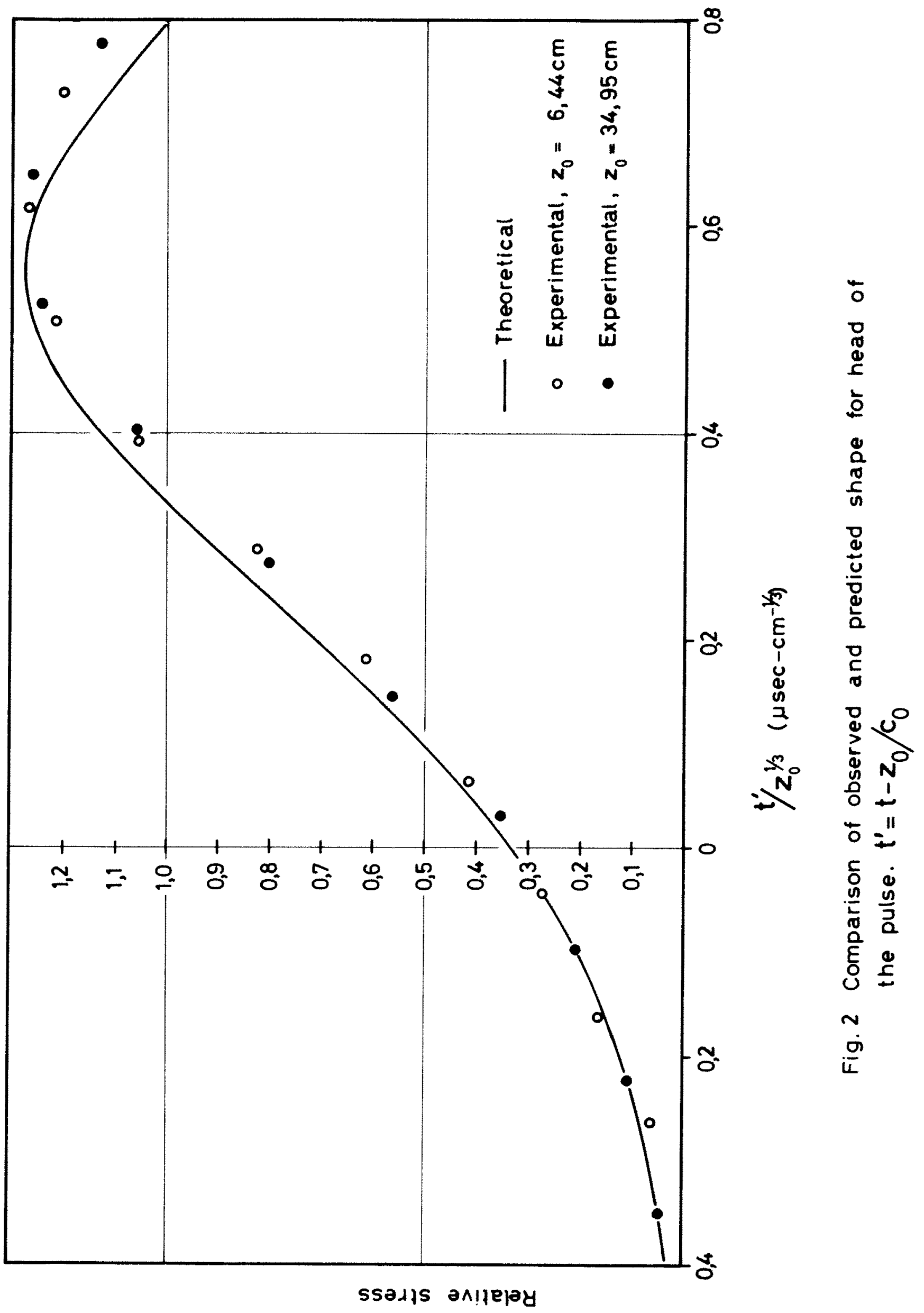




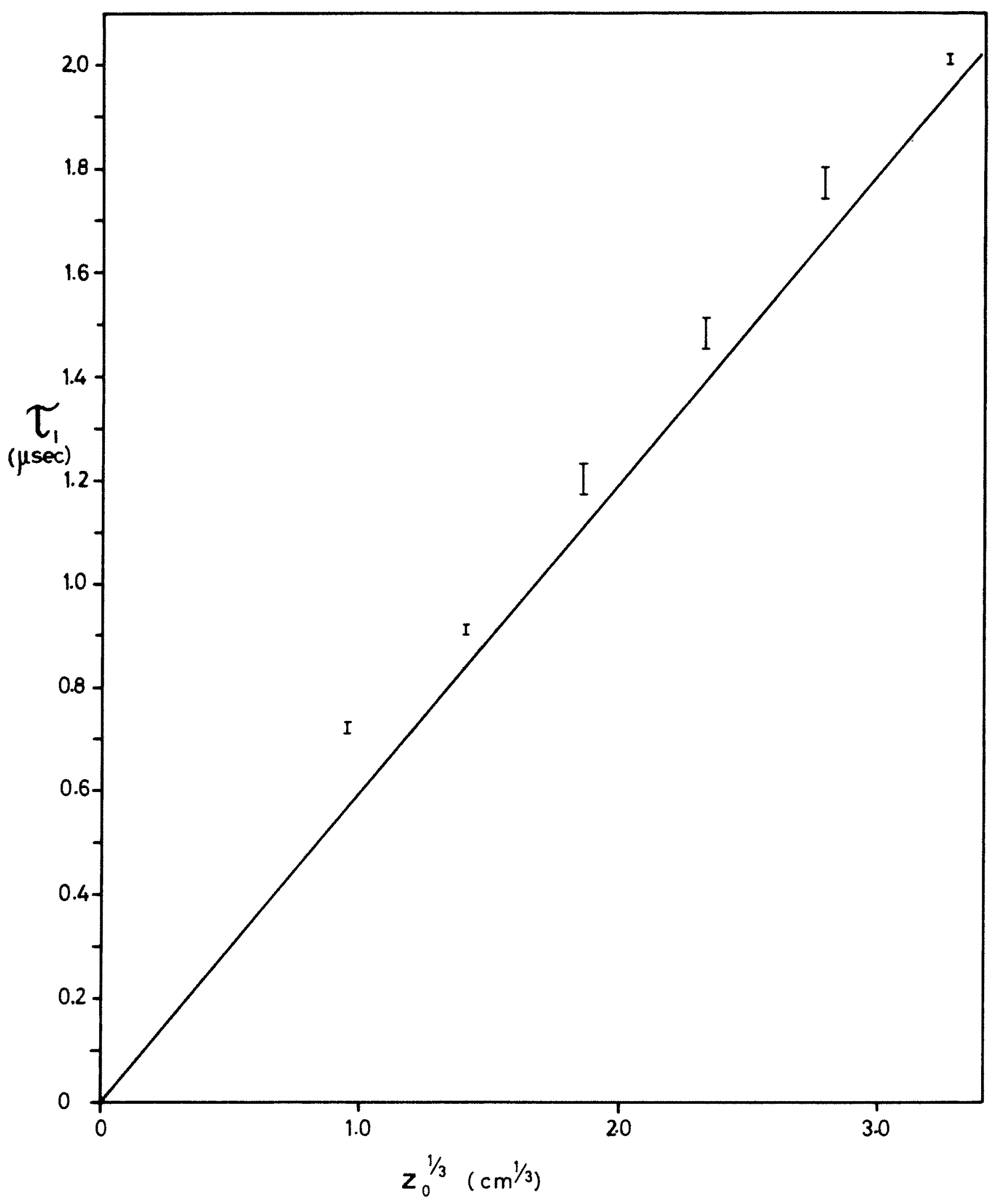

Fig. 3 Comparison of observed and predicted dependence of risetime on the distance, $\mathbf{z}_{0}$, of the observation point from the stressed end. 

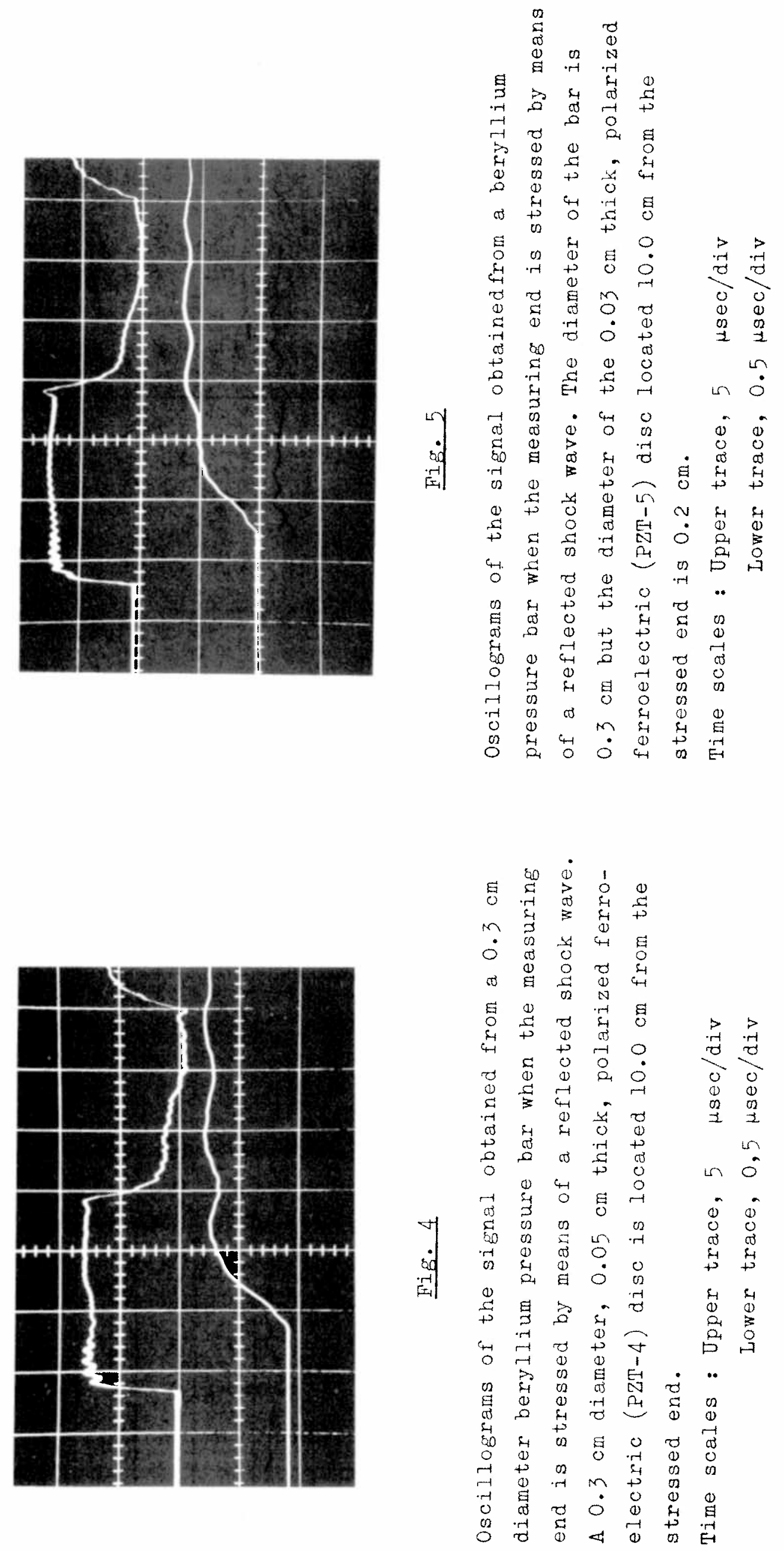


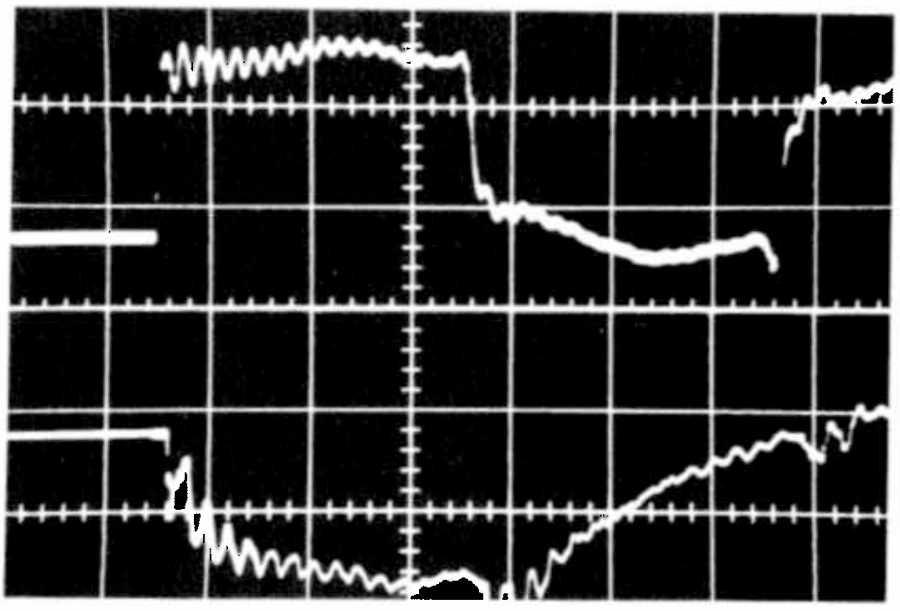

Fig. 6

Examples of output signal obtained from the beryllium pressure bar when the ferroelectric disc is bady cemented into the bar.

Time scales : Upper trace, $5 \mathrm{\mu sec} / \mathrm{div}$ Lower trace, $5 \mathrm{\mu sec} / \mathrm{div}$

(The signal polarity is reversed for the lower trace). 